\title{
Determinação do módulo de cisalhamento do Poliestireno Expandido por torção utilizando uma abordagem ortotrópica
}

\author{
Evaluation of the Expanded Polystyrene's shear \\ modulus by torsion using an orthotropic approach
}

Pedro Vitor de Barba ${ }^{1}$, Ricardo Pedro Bom ${ }^{2}$, Alexandre Wentz Zorzi ${ }^{1}$, Guilherme de Faveri ${ }^{1}$, Renato Barbieri ${ }^{2}$

\footnotetext{
${ }^{1}$ Programa de Pós-Graduação em Ciência e Engenharia de Materiais, PGCEM/UDESC, CEP: 89.219-710, Joinville, SC, Brasil.

2 Departamento de Engenharia Mecânica, UDESC, Universidade do Estado de Santa Catarina. Campus Universitário, CEP: 89219-710, Joinville, SC, Brasil.

e-mail: pedrodebarba@gmail.com, ricardo.bom@udesc.br, alexandrewentz@gmail.com, guilherme.faveri@gmail.com, renato.barbieri@udesc.br
}

\begin{abstract}
RESUMO
Neste artigo foi utilizado um sistema equações e aplicado um procedimento numérico para determinar, por meio de uma abordagem ortotrópica, o valor do módulo de cisalhamento do Poliestireno Expandido (EPS) a partir dos resultados experimentais de ensaios de torção em corpos de prova obtidos em três direções ortogonais. Os corpos de prova utilizados neste trabalho foram extraídos de um bloco de EPS utilizado na construção civil para a estabilização de solos moles denominados Geofoam. Em suas aplicações esses blocos estão sujeitos a esforços de compressão, tração e torção. A massa específica média do EPS foi de 26,51 $\pm 3,21$ $\mathrm{kg} / \mathrm{m}^{3}$ e suas pérolas maturadas tinham diâmetros que variavam entre 0,9 e $2,5 \mathrm{~mm}$. Os módulos de cisalhamento aparente, obtidos pelos ensaios de torção, alimentaram o sistema de equações não lineares proposto por Lekhnitskiy e Semenov desenvolvido para materiais ortotrópicos. Tal equacionamento foi resolvido através do método numérico de Newton-Raphson e todas as soluções possíveis foram apreciadas. Os resultados indicaram que os valores médios de $\mathrm{G}$ de cada plano podem ser representados pelo modelo ortotrópico, sendo as suas ordens de grandeza; $G_{x y}=6,101 \mathrm{MPa}, G_{x z}=5,796 \mathrm{MPa}$ e $G_{y z}=5,736 \mathrm{MPa}$.
\end{abstract}

Palavras-chave: Geofoam, ASTM E143-13, Anisotropia.

\begin{abstract}
In this paper an equations system was used and a numerical procedure was applied to determine, with an orthotropic approach, the shear modulus of Expanded Polystyrene (EPS) by experimental torsion tests using specimens from three orthogonal direction. The specimens used in this work were obtained from a EPS's block used in the construction for soft soil stabilization called Geofoam. In their applications these blocks are subjected to compression, tensile and torsion strengths. The mean EPS specific mass was $26.51 \pm 3.21 \mathrm{~kg} /$ $\mathrm{m}^{3}$ and its mature pearls had diameters ranging from 0.9 to $2.5 \mathrm{~mm}$. The apparent shear moduli, obtained by torsion tests, fed the nonlinear system proposed by Lekhnitskiy and Semenov and developed for orthotropic materials. Such equation was solved using the Newton-Raphson numerical method, all possible solutions were appreciated. The results indicated that the average values of $\mathrm{G}$ of each plane can be represented by the orthotropic model, thus their orders of magnitude; $\mathrm{G}_{\mathrm{xy}}=6,101 \mathrm{MPa}, \mathrm{G}_{\mathrm{xz}}=5,796 \mathrm{MPa}$ and $\mathrm{G}_{\mathrm{yz}}=5,736 \mathrm{MPa}$.
\end{abstract}

Keywords: Geofoam, ASTM E143-13, Anisotropy.

\section{INTRODUÇÃO}

O Poliestireno Expandido (EPS) é um material polimérico de natureza celular produzido a partir da expansão de pérolas de Poliestireno. O seu baixo custo de fabricação viabiliza a aplicação do EPS em diversas áreas 
como por exemplo; na indústria de embalagens [1], em artigos para esportes de alto rendimento [2], nos núcleos de painéis sanduíches utilizados na construção civil com finalidade estrutural e de isolamento térmico $[3,4]$ entre outros. Propriedades notáveis são atribuídas a esse material, tais como: baixo peso, baixa condutividade térmica, resistência à umidade, absorção acústica entre outras [5, 6].

Neste artigo foi ensaiado o EPS utilizado na construção civil denominado Geofoam. Estes blocos são usados para execução de aterros, construção de estradas e estabilização de encostas. Estudo realizado em rodovia, que empregaram o Geofoam na sua construção, avaliou a deformação sob compressão variando a absorção de água e o tipo de carregamento (estático e dinâmico) uma vez que para esta aplicação estava sujeita a esses esforços e intempéries [6].

Em estudos macro e microscópico, deformações por compressão, mostraram células de EPS movendo-se em diferentes direções ao carregamento aplicado. O que indicou que não somente esforços de compressão estavam atuando no experimento, mas também forças de cisalhamento [4, 7]. De maneira geral, não só com o EPS, são escassos os estudos sobre tensão de cisalhamento considerando a importância prática do tema $[8,9]$.

Quando as propriedades mecânicas de um material são diferentes em todas as direções, esse material é denominado anisotrópico. Existe uma forma particular de anisotropia no qual um material possui propriedade mecânica diferente nos três planos ortogonais, para esse caso dá-se o nome de ortotropia. Materiais celulares, como o EPS, na sua maioria são considerados anisotrópicos [10] devido principalmente ao processo de fabricação. Foi possível observar, em alguns estudos, cuidados tomados para que os corpos de prova fossem extraídos de uma mesma direção a fim de evitar divergências devido à anisotropia do EPS [6].

\section{MATERIAIS E MÉTODOS}

O Geofoam de EPS utilizado neste trabalho foi produzido pela empresa Knauf do Brasil Ltda. A massa especifica nominal fornecida pelo fabricante foi de $30 \mathrm{~kg} / \mathrm{m}^{3}$, já o valor médio e de dispersão obtidos experimentalmente (por meio da medição da massa e do volume aparente) foi de $26,51 \pm 3,21 \mathrm{~kg} / \mathrm{m}^{3}$. As pérolas maturadas, utilizadas para a expansão final, apresentaram diâmetros variando de 0,9 a 2,5 mm (dados fornecidos pelo fabricante).

Um bloco de EPS medindo 4090 x 1270 x $1050 \mathrm{~mm}$ foi cortado aleatoriamente para obter um bloco menor com dimensões de 575 × 225 x $445 \mathrm{~mm}$. Este por sua vez foi dividido novamente em três partes de onde foram extraídos 15 corpos de prova $(\mathrm{CP})$ de cada direção, perpendiculares entre si como indicado na Figura 1. Nesta figura foi indicado o sistema ortogonal X, Y e Z, utilizado como referência para a identificação dos planos de cisalhamento.

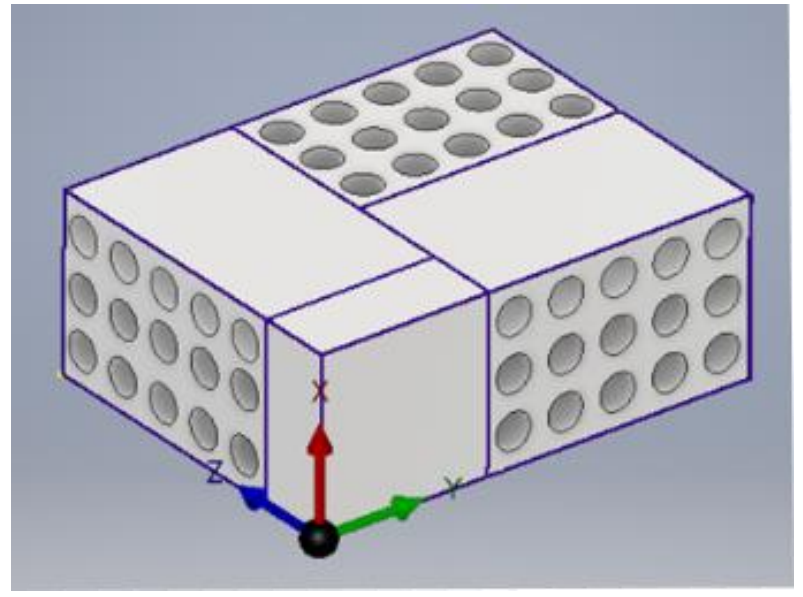

Figura 1: Sistema ortogonal de referência para o bloco de EPS e os corpos de prova. Fonte: Autor (2019).

Para obter os corpos de prova cilíndricos foi utilizado um dispositivo de filamento aquecido. Cada CP teve seu diâmetro $(D)$ e comprimento $(L)$ mensurado e registrado. Na média geral foi obtido um comprimento de 175,29 $\pm 0,25 \mathrm{~mm}$ e diâmetro de 47,04 $\pm 0,25 \mathrm{~mm}$.

A máquina de ensaio de torção utilizada tinha duas placas de três castanhas para a fixação do CP. Para que não danificassem o EPS, foram colados discos de aço nas extremidades de cada CP. Para obter a adesão, foi utilizada uma resina termorrígida do tipo epóxi, resina HEC 10 e endurecedor HEH 2000 (fabricante Hard 
Comércio de Fixadores e Resinas LTDA), solução essa também aplicada por VEJELIS et. al [4]. Após a união foi aguardado cinco dias a temperatura ambiente para a cura completa da resina.

Todos os ensaios realizados seguiram os procedimentos descritos na norma ASMT E143-13, [11]. Antes da realização dos ensaios, com a finalidade de estabelecer o equilíbrio térmico dos corpos de prova e do equipamento de ensaio, todos CP's foram mantidos em recinto fechado com temperatura controlado por 24 horas.

O equipamento utilizado foi do tipo home made melhor descrito por ESPINDOLA [12] cuja velocidade máxima de ensaio monotônico foi de $2 \mathrm{rpm}$, torque máximo de $10 \mathrm{Nm}$ e erro de controle no torque de $\pm 1 \%$ (Figura 2). A célula de carga utilizada foi do tipo S-10, com capacidade de carga de $100 \mathrm{~N}$, fabricante Alpha Instrumentos. O equipamento encontra-se no Laboratório de Polímeros da Universidade do Estado de Santa Catarina.

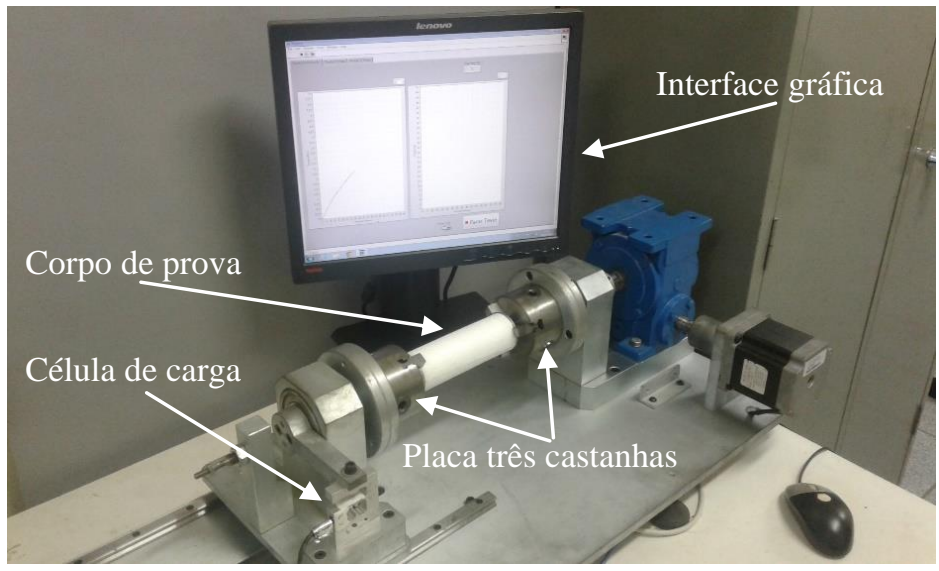

Figura 2: Aparato experimental. Fonte: Autor (2019).

O valor do módulo de cisalhamento depende da direção em que foi medido no material, se o mesmo apresentar comportamento ortotrópico. Para corpos de prova com geometria cilíndrica, o módulo de cisalhamento aparente é dado pela Equação 1 [11]:

$$
G_{a p}=\frac{32 \times L \times T}{\pi \times D^{4} \times \phi}
$$

Onde, $G_{a p}$ é o módulo de cisalhamento aparente (MPa), $T$ é o Torque (Nmm), $L$ é o comprimento do corpo de prova (mm), $D$ o diâmetro do corpo de prova $(\mathrm{mm})$ e $\phi$ é o ângulo de torção (radianos).

A Figura 3a mostra uma curva $T_{y}$ vs. $\phi_{y}$ típica para os CP's ensaiados. O valor de $G_{a p}$ foi determinado multiplicando o coeficiente angular da reta ajustada $(\mathrm{f}(\mathrm{x})=16101 \mathrm{x}-19,887$ para o $\mathrm{CP} 17)$ por $32 L / \pi D^{4}$. Quando essa reta foi representada graficamente em escala logarítmica foi possível observar a sensibilidade do ajuste feito (Figura 3b). 


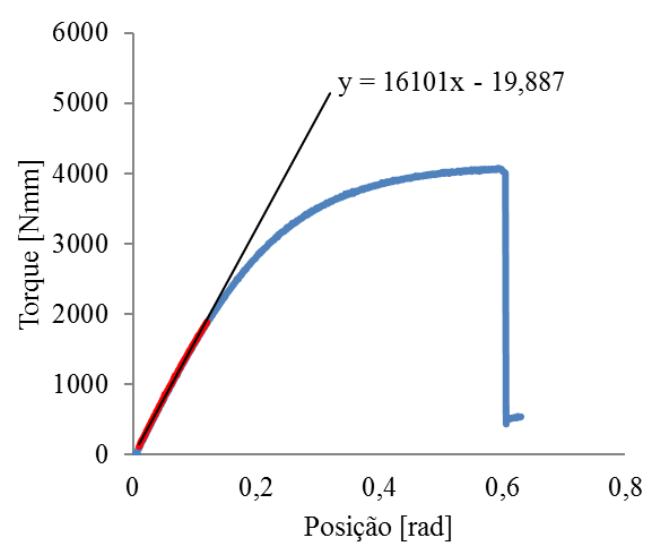

a)

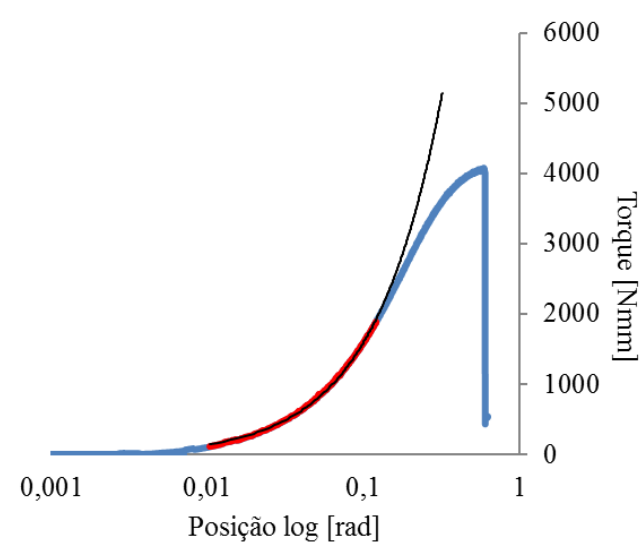

b)

Figura 3: Em a) curva típica para Ty vs. фy, CP 17 (plano xz) e em b) detalhe da sensibilidade da reta interpolada. Fonte: Autor (2019).

A reta interpolada, por regressão linear, foi determinada em planilha eletrônica programada para desconsiderar os 100 primeiros pontos coletados e selecionar o intervalo de dados que apresenta $\mathrm{R}^{2}>0,999$. De maneira análoga foram determinados os valores para $2 G_{x y} G_{x z} /\left(G_{x y}+G_{x z}\right)$ e $2 G_{x z} G_{y z} /\left(G_{x z}+G_{y z}\right)$ com os ensaios nas direções x e $\mathrm{z}$, respectivamente.

Quando um material apresentar comportamento anisotrópico uma aproximação que pode ser feita é considerar o comportamento ortotrópico, assim, um modelo utilizado para caracterizar o mesmo é descrito pela Equação 2, [13, 14]:

$$
2 \times\left(\frac{G_{x y} G_{x z}}{G_{x y}+G_{x z}}\right)=\frac{32 \times L \times T_{x}}{\pi \times D^{4} \times \phi_{x}}
$$

Onde; $G_{x y}$ indica o módulo de cisalhamento no plano $x y(\mathrm{MPa}), G_{x z}$ representa o módulo de cisalhamento no plano $x z(\mathrm{MPa}), \phi_{x}$ designa o ângulo de torção na direção $x$ (radianos), $T_{x}$ indica o torque na direção $x(\mathrm{Nmm})$.

Se $G_{x y}=G_{x z}$ a Equação 2, volta a condição da Equação 1, como esperado. Derivando $T_{x}$ em relação a $\phi_{x}$ resulta na Equação 3, tem-se:

$$
\frac{2 G_{x y} G_{x z}}{G_{x y}+G_{x z}}=\frac{d T_{X}}{d \phi_{X}} \frac{L \times 32}{\pi \times D^{4}}
$$

Expressões análogas à esta podem ser escritas para as demais direções ortogonais ao plano cartesiano, isto é, Equações 4 e 5:

$$
\begin{aligned}
& \frac{2 G_{x y} G_{y z}}{G_{x y}+G_{y z}}=\frac{d T_{y}}{d \phi_{y}} \frac{L \times 32}{\pi \times D^{4}} \\
& \frac{2 G_{x z} G_{y z}}{G_{x z}+G_{y z}}=\frac{d T_{z}}{d \phi_{z}} \frac{L \times 32}{\pi \times D^{4}}
\end{aligned}
$$

Apesar do modelo proposto por LEKHNITSKIY [13] e SEMENOV [14] não fazer menção sobre aplicações em materiais celulares, este foi empregado nesse trabalho.

Após a estimativa do módulo de cisalhamento aparente, o lado direito das Equações (3), (4) e (5) foram conhecidos e o sistema de equações não lineares pode ser resolvido simultaneamente para determinar os parâmetros do material $G_{x y}, G_{x z}$ e $G_{y z}$.

Com a finalidade de contemplar todos os valores possíveis, todas as combinações de $G_{a p x,} G_{\text {apy }}$ e $G_{\text {apz }}$ 
foram realizadas. Os resultados desta etapa foram obtidos utilizando o método numérico de Newton Raphson para múltiplas variáveis por meio de aplicativo ${ }^{\text {TMMatLab [15]. }}$

\section{RESULTADOS}

Em todos os ensaios de torção realizados, o padrão de falha na ruptura do CP foi de $45^{\circ}$ em relação ao eixo de torção (Figura 4a e 4b). Todas as fraturas ocorreram de tal maneira que mantiveram a estrutura das pérolas expandidas intactas, ou seja, uma fratura intercelular por descolamento das interfaces (Figura 4c).

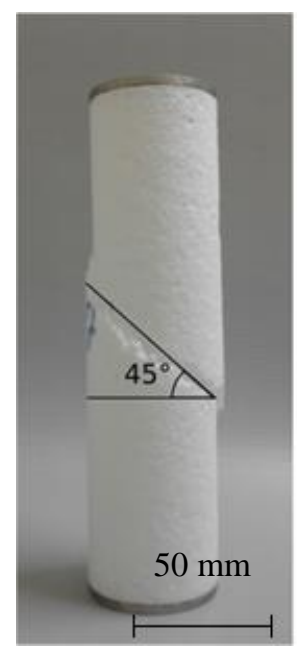

a)

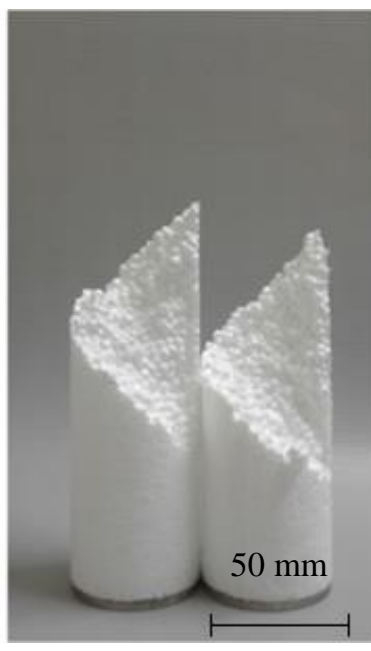

b)

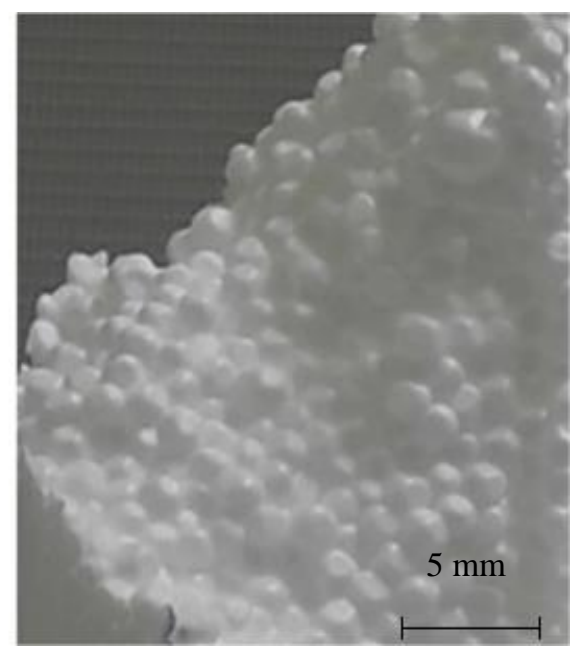

c)

Figura 4: a) e b) fratura em $45^{\circ}$ do CP 17. c) Aproximação expondo fratura intercelular. Fonte: Autor (2019).

Quando representadas lado a lado, as curvas Torque vs. Ângulo de torção, de cinco CP's de cada direção, foi possível observar um comportamento comum para cada direção, porém, diferente entre as direções (Figura 5).
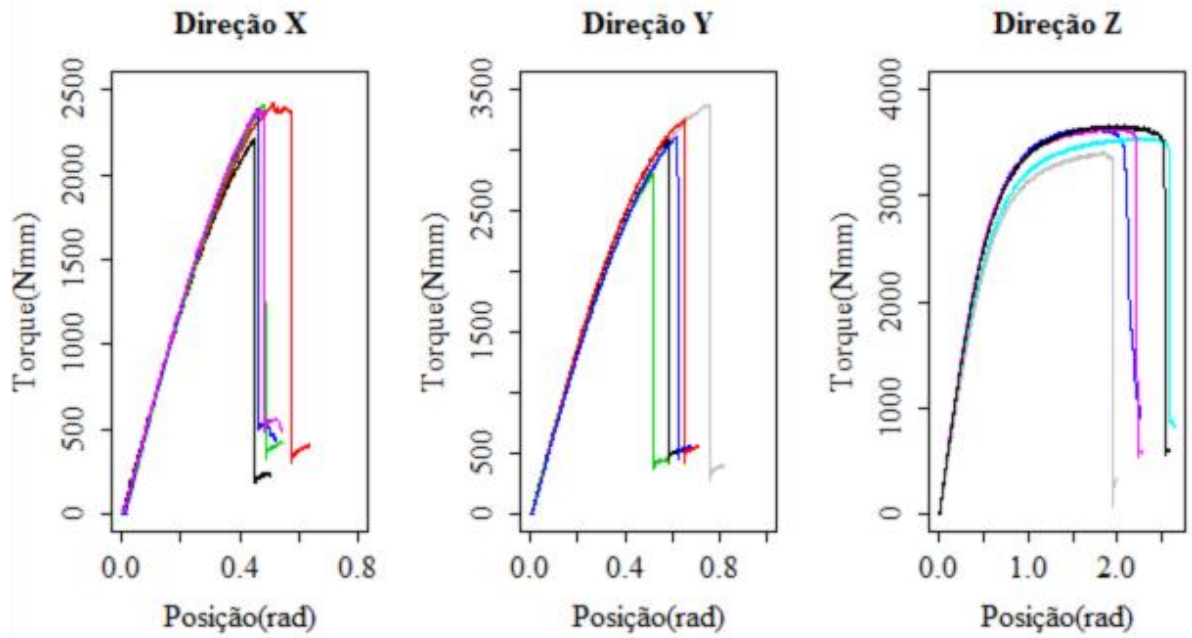

Figura 5: Gráficos Torque vs. Ângulo de torção para as três direções. Fonte: Autor (2019).

Da Figura 5 acima pode-se observar que para as diferentes direções os torques máximos e os ângulos de torção máximos foram diferentes. Já a direção $\mathrm{Z}$ suportou um maior alongamento, por consequência os CP's dessa direção foram mais dúcteis que os demais. Tal discrepância já seria suficiente para verificar a anisotropia do material.

Entre as principais suposições que poderiam explicar esse comportamento estão: o direcionamento do 
fluxo de vapor dentro do molde, este vapor pode aquecer as pérolas maturadas de maneira diferenciada, o que permitiu uma melhor adesão nas partes das faces mais aquecidas; com relação ao gradiente de temperatura dentro do molde entre a entrada e saída de vapor, possivelmente as forças de adesão das pérolas que estão mais próximo da entrada de vapor, sejam maiores do que entre aquelas pérolas que estão mais afastadas.

Os módulos de cisalhamento aparente $\left(G_{a p}\right)$ obtido pela Equação (1) para os 15 corpos de prova em cada direção estão representados na Tabela 1.

Tabela 1: Módulos de cisalhamento aparente obtidos nos ensaios de torção nas três direções. Fonte: Autor (2019).

\begin{tabular}{ccc}
\hline $\boldsymbol{G}_{a p x}(\mathbf{M P a})$ & $\boldsymbol{G}_{a p y}(\mathbf{M P a})$ & $\boldsymbol{G}_{a p z}(\mathbf{M P a})$ \\
\hline 6,086 & 5,922 & 6,174 \\
5,359 & 6,276 & 6,263 \\
5,920 & 6,190 & 6,384 \\
5,809 & 6,442 & 6,021 \\
5,584 & 6,469 & 6,113 \\
5,800 & 5,444 & 5,754 \\
5,837 & 5,493 & 5,689 \\
5,785 & 6,277 & 6,102 \\
5,722 & 6,374 & 5,796 \\
5,550 & 6,152 & 5,765 \\
5,988 & 4,871 & 5,092 \\
5,975 & 5,202 & 5,049 \\
5,862 & 5,758 & 5,047 \\
5,763 & 6,256 & 5,219 \\
5,797 & 6,165 & 5,236 \\
\hline
\end{tabular}

A solução do sistema de equações não lineares, Equações 3 a 5, montada a partir dos resultados da Tabela 1, forneceu uma população de $G_{x y}, G_{x z}$ e $G_{y z}$ para cada um dos 3375 arranjos possíveis.

Como os desvios padrão populacionais de cada plano de cisalhamento são conhecidos $\left(\sigma_{x y}=0,788 \mathrm{MPa}, \sigma_{\mathrm{xz}}=0,705 \mathrm{MPa}\right.$ e $\left.\sigma_{\mathrm{yz}}=0,651 \mathrm{MPa}\right)$ e atribuindo um erro $(\varepsilon)$ de $0,263 \mathrm{MPa}$ [8], com nível de confiança $(\alpha)$ de $99 \%$ foi utilizado a Equação 6 que permitiu determinar o tamanho da amostra [16]:

$$
n=\frac{\sigma^{2} Z_{\alpha / 2}^{2}}{\varepsilon^{2}}
$$

Onde, $\sigma$ representa o desvio padrão populacional, $Z_{\alpha / 2}$ é o valor crítico que corresponde ao grau de confiança desejado e $\varepsilon$ a margem de erro. Assim, foram retiradas amostras aleatórias de tamanhos $n_{x y}=60$, $n_{x z}=48$ e $n_{y z}=41$ de direção.

Com o pacote de funções RriskDistribution do aplicativo $\mathrm{R}^{\mathrm{TM}}$, uma vez inserido os dados de uma distribuição o pacote ranqueia, entre as distribuições mais conhecidas, a que melhor se ajusta aos dados fornecidos. Assim, foi estabelecido que a distribuição normal foi a distribuição que melhor representou os dados de cada uma das três amostras. Foram calculados a média e o desvio padrão $\left(G_{x y}=5,862 \pm 0,815 \mathrm{MPa}, G_{x z}=5,541 \pm 0,760 \mathrm{MPa}\right.$ e $\left.G_{y z}=5,329 \pm 0,649 \mathrm{MPa}\right)$. Na Figura 6 observa-se o histograma de cada amostra e a sua distribuição normal, representada pela linha contínua.

Para o plano de cisalhamento $G_{x y}, 16,67 \%$ das observações puderam ser consideradas iguais para um intervalo de confiança de $95 \%$ (linha tracejada na Figura 6). Analogamente 27,08\% para $G_{x z}$ e $14,63 \%$ para $G_{y z}$. Ou seja, o módulo de cisalhamento do EPS, com uma abordagem ortotrópica, apresentou comportamento não homogêneo e, portanto, na média, pôde ser representado pelo modelo ortotrópico. 

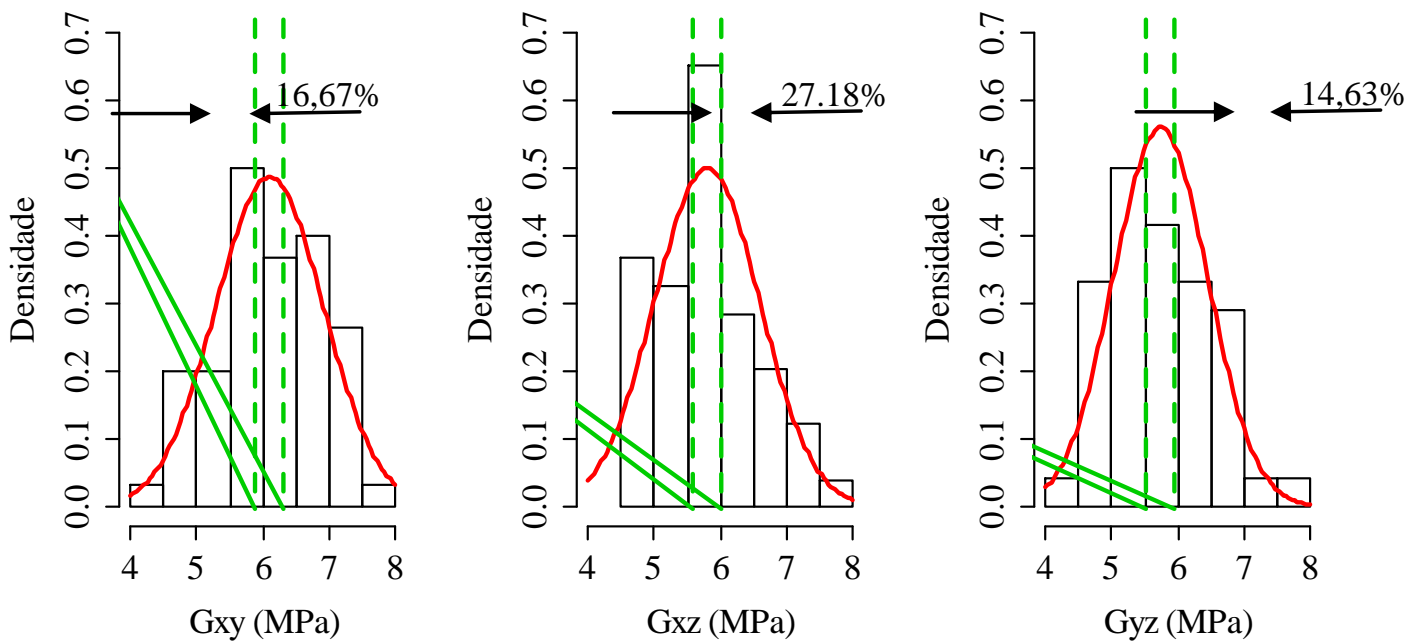

Figura 6: Distribuições das amostras em função dos planos de cisalhamento.

Para comprovar estatisticamente a diferença no módulo de cisalhamento entre os três planos, foi aplicado o teste estatístico de análise de variância (ANOVA). Para isso as distribuições dos módulos respeitaram os três requisitos para aplicação da ANOVA; observações independentes, homogeneidade da variância entre os módulos e resíduos com distribuição normal. A Tabela 2 mostra a análise de variância, cuja hipótese nula foi rejeitada, logo os três planos de cisalhamento apresentaram valores diferentes entre si para o módulo de cisalhamento em uma abordagem ortotrópica.

Tabela 2: Análise de Variância (ANOVA). Fonte: Autor (2019)

\begin{tabular}{|c|c|c|c|c|c|}
\hline & $\begin{array}{c}\text { GRAU } \\
\text { DE LIBERDADE }\end{array}$ & $\begin{array}{c}\text { SOMA } \\
\text { DOS QUADRADOS }\end{array}$ & $\begin{array}{l}\text { QUADRADO } \\
\text { MÉDIO }\end{array}$ & ESTATÍSTICA F & P-VALOR \\
\hline Planos & 2 & 7,27 & 3,634 & 6,253 & 0,00248 \\
\hline Resíduos & 148 & 84,85 & 0,581 & & \\
\hline
\end{tabular}

Assim, os valores dos módulos de cisalhamento em uma abordagem ortotrópica são representados pela esperança da distribuição de cada amostra; $G_{x y}=6,101 \pm 0,812 \mathrm{MPa}, G_{x z}=5,796 \pm 0,788 \mathrm{MPa}$ e $G_{y z}=5,736 \pm 0,704 \mathrm{MPa}$.

\section{DISCUSSÃO}

O resultado obtido nesse trabalho possui a mesma ordem de grandeza registrada TALWIN [9], porém, naquele estudo não foi mencionado como o valor foi obtido e nem qual modelo foi adotado. Tal resultado pode explicar alguns problemas encontrados na aplicação do EPS, como por exemplo, em empilhamentos de embalagens que utilizam EPS para proteção de produtos. Esse resultado também se aplica como ponto de atenção para desenvolvedores de soluções com EPS, uma vez que houve diferença do módulo de cisalhamento segundo a direção.

\section{CONCLUSÕES}

Os procedimentos descritos nesse artigo se mostraram eficazes para verificar a não homogeneidade do módulo de cisalhamento do EPS utilizando uma abordagem estatística, uma vez que as amostras extraídas, de cada direção, não convergiram para um único valor central do módulo de cisalhamento. No plano de cisalhamento que melhor representou uma tendência central apenas $27,08 \%$ das observações poderiam ser consideradas iguais. Logo, o módulo de cisalhamento, não se mostrou homogêneo para cada direção.

O comportamento ortotrópico foi verificado estatisticamente pela aplicação da análise de variância (ANOVA) cuja hipótese nula (módulo de cisalhamento igual entre as direções) foi rejeitada. Logo, a aproximação realizada pôde ser comprovada.

As amostras apresentaram uma distribuição normal em todos os planos. Os parâmetros que melhor as 
representaram foram suas respectivas médias e desvios padrão. Os valores encontrados para os módulos de cisalhamento do EPS de massa especifica medida de $26,51 \pm 3,21 \mathrm{~kg} / \mathrm{m}^{3}$ foram $G_{x y}=6,101 \pm 0,812 \mathrm{MPa}$, $G_{x z}=5,796 \pm 0,788 \mathrm{MPa}$ e $G_{y z}=5,736 \pm 0,704 \mathrm{MPa}$. Portanto, o modelo ortotrópico pode ser representado pela média dos módulos de cisalhamento obtidos a partir das três direções.

\section{BIBLIOGRAFIA}

[1] SONG, B.. CHEN, W.W., DOU, S., et al., "Strain-rate effects on elastic and early cell-collapse responses of a polystyrene foam," Int. J. Impact Eng., v. 31, n. 5, pp. 509-521, Fev. 2005.

[2] DI LANDRO, L., SALA, G., OLIVIERI, D. "Deformation mechanisms and energy absorption of polystyrene foams for protective helmets," Polymer Testing, v. 21, n. 2, pp. 217-228, Fev. 2002.

[3] SMAKOSZ, T., TEJCHMAN, J. "Evaluation of strength, deformability and failure mode of composite structural insulated panels," Mateials and Design, v. 54, pp. 1068-1082, Jan. 2014.

[4] VEJELIS, S., GNIP, I., VAITKUS, S., et al., "Shear strength and modulus of elasticity of expanded polystyrene (EPS)," Material Science (Medziagotyra), v. 14, n. 3, pp. 230-233, Jan 2008.

[5] HORVATH, J.S. "Expanded Polystyrene (EPS) geofoam: An introduction to material behavior," Geotextiles and Geomembranes, v. 13, n. 4, pp. 263-280, Jan 1994.

[6] DUSKOV, M. "Materials research on EPS20 and EPS15 under representative conditions in pavement structures," Geotextiles and Geomembranes, v. 15, n. 1-3, pp. 147-181, Jan. 1997.

[7] VAITKUS, S., LAUKAITIS, A., GNIPAS, I., et al. JELIs, "Experimental Analysis of Structure and Deformation Mechanisms of Expanded Polystyrene ( EPS ) Slabs", Material Science (Medziagotyra), v. 12, n. 4, pp. 323-327, Fev. 2006.

[8] GNIP, I.J., VEYELIS, S.A., KERSULIS, V.I., et al. "Deformability and strength of expanded polystrene (EPS) under short-term shear loading", Mechanics of Composite Materials, v. 43, n. 1, pp. 85-94, Jan 2007.

[9] TALWIN, H. Development of a Method to Analyze Structural Insulated Panels Under Transverse Loading, Tese de M.Sc, Departament of Civil and Envrionmental Engineering/Washington Estate University, Pullman, Washington, Estados Unidos, Dez. 2002.

[10] GIBSON, L.J., ASHBY, M.F. Cellular solids: Structure and properties, 2 ed., Reino Unido, Cambridge University Press, 1997.

[11] ASTM E143-13, "Standard Test Method for Shear Modulus at Room Temperature 1," American Society for Testing and Materials, pp. 1-5, Fev. 2014.

[12] ESPINDOLA, G. Projeto de uma máquina para ensaios de torção em baixa frequência, Tese de M.Sc, PGCEM/UDESC, Joinville, Santa Catarina, Brasil, Fev. 2015.

[13] LEKHNITSKIY, S.G. Anisotropic Plates, 1 ed., Estados Unidos, Foreign Technology Division, 1969.

[14] SEMENOV, P.I. "Determination of shear moduli of orthotropic materials from torsion tests," Polymers Mechanics., v. 2, n. 1, pp. 17-20, Fev. 1968.

[15] THE MATHWORKS INC., "MATLAB (R2015a)," The MathWorks Inc. 2015.

[16] MONTGOMERY, D.C., RUNGER, G.C., Applied Statistics and Probability for Engineers, 5 ed., Estados Unidos, John Wiley \& Sons, Fev. 2011.

\section{ORCID}

Pedro Vitor de Barba

Ricardo Pedro Bom

Alexandre Wentz Zorzi

Guilherme de Faveri

Renato Barbieri https://orcid.org/0000-0003-1158-2707

https://orcid.org/0000-0002-3235-3041

https://orcid.org/0000-0002-6624-664X

https://orcid.org/0000-0001-9004-7846

https://orcid.org/0000-0001-7180-1014 